\title{
MEDICALLY IMPORTANT COAGULASE NEGATIVE STAPHYLOCOCCI
}

\author{
NagarajuVanaparti ${ }^{1}$, Kalpana Sadawarte ${ }^{2}$
}

${ }^{I}$ PhD Scholar, ${ }^{2}$ Professor and Head, Microbiology, People's University, Bhanpur, Bhopal, Madhya Pradesh

\begin{abstract}
In recent years, coagulase negative Staphylococci (CONS) are increasingly recognized as one of the major causes of health care associated infections (HCAI). CONS infections are one of the major causes of morbidity and mortality in hospitalized patients. As CONS colonize various parts of the skin and mucous membranes, most of infections are endogenous in origin. However, they are also transmitted exogenously via various medical/ surgical procedures. The aim of present review article is to update and summarize the information available on medically important CONS with special reference to epidemiology, laboratory diagnosis and antibiotic resistance.
\end{abstract}

Keywords: Coagulase negative Staphylococci; Biofilm formation; Exoenzymes; Methicillin resistance

\section{INTRODUCTION}

In recent years, coagulase negative Staphylococci (CONS) are increasingly recognized as one of the major causes of health care associated infections (HCAI). CONS infections are one of the major causes of morbidity and mortality in hospitalized patients [1]. CONS infections especially bacteremia contributes to $12-80 \%$ of mortality in hospitalized patients [1]. Developments in the field of medicine, multi-morbid and immunocompromised status of the host and the increased usage of inserted and implanted medical devices are important factors contributing to the emergence of CONS infections [2].

CONS represent a heterogeneous group within genus Staphylococcus. Historically, isolation of these organisms was ignored as culture contaminants or nonpathogenic isolates. Kloos and Bannerman (1994) provided the update on the clinical significance of CONS [3]. CONS can cause various afflictions in both immunocompetent and immunocompromised individuals. Endocarditis central nervous system (CNS) shunt infections are usually seen in immunocompromised hosts and patients with indwelling medical devices whereas, urinary tract infection is the most CONS infection reported in healthy individuals [4].

As CONS colonize various parts of the skin and mucous membranes, most of infections are endogenous in origin. However, they are also transmitted exogenously

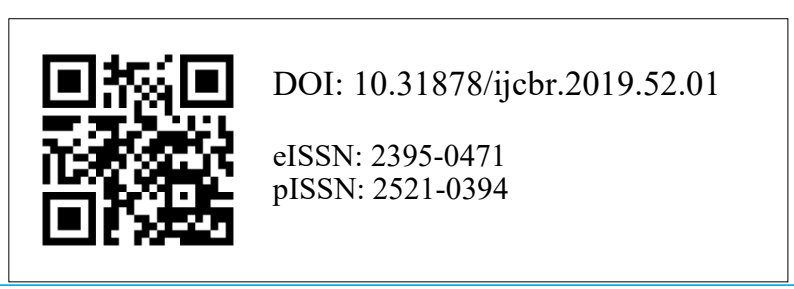

via various medical/ surgical procedures. Among CONS, S. epidemidis, S. saprophyticus and S. haemolyticus are quite widely recognized as cause of human infections [2]. Other species like S. warneri, S. hominis and $S$. simulans are considered as emerging [2].

Furthermore, National Nosocomial infection Survey (NNIS) conducted between 1980 to 1989 , reported 20 to $60 \%$ CONS isolates to be resistant to methicillin, oxacillin or nafcillin [5]. Multidrug resistant CONS also often colonize the skin of health-care workers and hospitalized patients [6]. Moreover, colonizing isolates often serve as a potential reservoir for antibiotic resistance genes that cannot only transfer among other CONS species but also can be acquired by S. aureus [7].

The aim of present review article is to update and summarize the information available on medically important CONS with special reference to epidemiology, laboratory diagnosis and antibiotic resistance.

Methodology: For preparation of this review, search was made on various web search engines like PubMed, and Google Scholar by using appropriate MeSH terms like Coagulase negative Staphylococci, CONS infections, CONS epidemiology and antibiotic resistance in CONS. Relevant literature were retrieved from these searches and used for preparation of the present review article.

General characteristics and classification of CONS: CoNS are included in family Micrococcaceae and genus Staphylococcus. Till recent, there 47 recognized species and 23 subspecies of Staphylococci [2]. The genus Staphylococci contains 39 species that fulfill the categorization of CONS [4]. Traditionally, Staphylococcal

Correspondence: Nagaraju V, Ph.D Scholar, Department of Microbiology, People’s University, Bhanpur, Bhopal, Madhya Pradesh. Email:vnr2275@gmail.com 
isolates that don't produce coagulase and thermo nuclease are termed as CONS [8].

In humans, CONS are regular microbiota of skin and mucous membrane. This group of organisms prefers human anatomical sites of high humidity like axillae, the gluteal and inguinal regions, the umbilicus, the anterior nares, the conjunctiva, the ocular spaces, the antecubital and popliteal spaces and plantar region of foot $[9,10]$. Table 1 shows the anatomical sites colonized by different CONS.

Table 1: Human anatomical sites colonized by different coagulase negative Staphylococci [2].

\begin{tabular}{l|l}
\hline CONS spp. & $\begin{array}{l}\text { Anatomical sites colo- } \\
\text { nized }\end{array}$ \\
\hline S. auricularis & External auditory canal \\
\hline $\begin{array}{l}\text { S. capitis subsp. capitis } \\
\text { S. capitis subsp. capitis }\end{array}$ & $\begin{array}{l}\text { Predominant in the scalp } \\
\text { and armsMostly on skin }\end{array}$ \\
\hline S. caprae & Skin, anterior nares \\
\hline S. cohnii subsp. cohnii & Skin \\
\hline $\begin{array}{l}\text { S. cohnii subsp. urealyti- } \\
\text { cus }\end{array}$ & Skin \\
\hline S. epidermidis & Skin \\
\hline S. haemolyticus & Skin \\
\hline S. lugdunensis & Skin \\
\hline S. saccharolyticus & Skin (forehead and skin) \\
\hline S. sciuri & Skin \\
\hline S. warneri & $\begin{array}{l}\text { Skin (nares, head, legs, } \\
\text { and arms) }\end{array}$ \\
\hline S. xylosus & Skin \\
\hline
\end{tabular}

CONS species found in clinical specimen can further classified on the basis of novobiocin resistance (Figure 1) $[11]$.

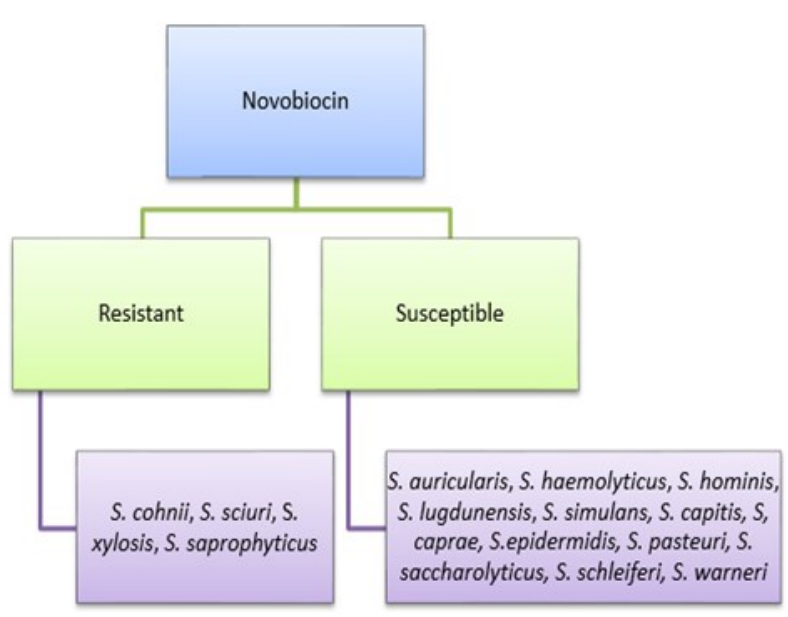

Fig 1: Classification of CONS on basis of novobiocin resistance.
Baird-Parker system was used for classification of CONS before 1975 [4]. In this system anaerobic glucose fermentation test was used to differentiate genus Staphylococcus from micrococcus [12]. Six groups were defined within the genus Staphylococcus by means of a number of biochemical and physiological characters. Staphylococcus subgroup I correspond to $S$. aureus and no specific names were assigned to subgroup II to VI [12]. After few years, sub group II-VI were changed to biotype I-V under the heading $S$. epidermidis. BairdParker's system for classification of Staphylococci and Micrococci is shown table 2 [12].

Table 2: Baird-Parker's system for classification of Staphylococci and Micrococci 2 [2].

\begin{tabular}{l|l|l|l|l|l|l}
\hline \multirow{2}{*}{ Character } & \multicolumn{5}{|l}{ Subgroup } \\
\cline { 2 - 7 } & I & II & III & IV & V & VI \\
\hline Coagulase & + & - & - & - & - & - \\
\hline Phosphatase & + & + & + & - & - & - \\
\hline $\begin{array}{l}\text { Acid from manni- } \\
\text { tol } \\
\begin{array}{l}\text { Aerobically } \\
\text { Anaerobically }\end{array}\end{array}+^{*}$ & - & - & - & - & - & + \\
\hline Acetoin & $+*$ & $+*$ & - & + & + & + \\
\hline $\begin{array}{l}\text { Acid from } \\
\text { Lactose } \\
\text { Maltose }\end{array}$ & $+*$ & $+*$ & V & - & + & V \\
\hline Growth at $10^{\circ} \mathrm{C}$ & $+*$ & - & - & - & - & $-*$ \\
\hline
\end{tabular}

\section{1: Staphylococcus}

‘*' Usual result, '+' negative, 'V' variable result

\begin{tabular}{|c|c|c|c|c|c|c|c|c|}
\hline \multirow{3}{*}{ Character } & \multicolumn{8}{|c|}{ Subgroup } \\
\hline & \multicolumn{4}{|c|}{ S. saprophyticus } & \multicolumn{4}{|c|}{ Micrococci } \\
\hline & 1 & 2 & 3 & 4 & 5 & 6 & 7 & 8 \\
\hline $\begin{array}{l}\text { Acid from } \\
\text { glucose } \\
\text { aerobically }\end{array}$ & + & + & + & + & + & + & - & - \\
\hline Phosphatase & - & - & - & - & - & + & - & - \\
\hline Acetoin & + & + & + & + & - & - & - & - \\
\hline $\begin{array}{l}\text { Terminal } \\
\text { pH, glucose } \\
\text { broth }\end{array}$ & $\begin{array}{l}4 . \\
6\end{array}$ & 5.1 & 5 & $\begin{array}{l}5 . \\
2\end{array}$ & 5.5 & 5.3 & $\begin{array}{l}6 . \\
5\end{array}$ & $\begin{array}{l}6 . \\
2\end{array}$ \\
\hline $\begin{array}{l}\text { Acid from } \\
\text { Arabinose } \\
\text { Lactose } \\
\text { Maltose } \\
\text { Mannitol }\end{array}$ & $\begin{array}{l}- \\
- \\
\text { V } \\
-\end{array}$ & $\begin{array}{l}- \\
+ \\
+ \\
-\end{array}$ & $\begin{array}{l}- \\
\mathrm{V} \\
+ \\
* \\
+\end{array}$ & $\begin{array}{l}+ \\
+ \\
+ \\
+\end{array}$ & $\begin{array}{l}\mathrm{V} \\
+^{*} \\
+^{*} \\
+^{*}\end{array}$ & $\begin{array}{l}+ \\
+ \\
+ \\
+\end{array}$ & $\begin{array}{l}- \\
- \\
- \\
-\end{array}$ & $\begin{array}{l}- \\
- \\
- \\
-\end{array}$ \\
\hline Lipolysis & $\mathrm{V}$ & + & + & - & $-*$ & $+*$ & $\mathrm{~V}$ & - \\
\hline $\begin{array}{l}\text { Tween hy- } \\
\text { drolysis }\end{array}$ & $-*$ & - & $\mathrm{V}$ & - & - & + & $\mathrm{V}$ & $\mathrm{V}$ \\
\hline $\begin{array}{l}\text { Growth at } \\
10^{\circ} \mathrm{C}\end{array}$ & $-*$ & $+*$ & $\begin{array}{l}+ \\
*\end{array}$ & + & $+^{*}$ & + & $+*$ & + \\
\hline Red pigment & - & - & - & - & - & - & - & + \\
\hline
\end{tabular}




\section{2: Micrococci}

‘*' Usual result, '+' negative, 'V' variable result

In 1975, a scheme based on 13 important features, like coagulase production, haemolysis, nitrate reduction and carbohydrate assimilation were introduced for biotyping of CONS [4]. CONS are generally oxidase negative but species like $S$. sciuri and $S$. fleurettii are oxidase positive. Some laboratories still prefer Baird-parker Scheme for identification of CONS isolates.

In recent years, genotypic methods based on 16s rRNA, sodA, gap, rpo $\mathrm{B}$, tuf gene sequencing have been developed for classification of CONS [13-16]. Commercial rapid identification systems for classification of CONS include API staph ID test, BD Phoenix system, VITEK 2 ID-GP. Genotypic methods are considered to be superior over phenotypic methods for identification of CONS, but certain phenotypic methods are routinely employed [4].

CONS involved in human infections: In 1980s, a wide range of infections were believed to be caused by CoNS, especially S. epidermidis (Mack,1990). However, Quinet al (1966) were first to notice the clinical importance of CONS.

CONS are embroiled in various infections like wound, urogenital tract, respiratory and central-nervous system (CNS) and blood stream infections [17]. Infections due CONS occurs when there is breach in natural mucocutaneous barrier and presence of transient or permanent indwelling medical devices.

CONS infections usually occur due to translocation of endogenous strain to anatomically sterile site mostly due to presence of indwelling-medical device. These group of organisms cause fatal infections in high risk neonates like neonates with poor perinatal history, premature new born, new born with invasive procedure like umbilical arterial/ venous catheterization, endotracheal intubation, jugular or subclavian central alimentation catheter etc.

S. epidermidis, S. haemolyticusandS. hominis are the most commonly isolated CONS species from human infections [4]. S. epidermidis is implicated in nosocomial blood stream infections, endocarditis, urinary tract and surgical site infections, central nervous system shunt infections, eye infections, intravascular catheterrelated infections, peritoneal dialysis-related infections and prosthetic joint infections $[18,4]$. S. epidermidis has become the most important model microorganism for studying device associated health-care associated infections [2]. This CONS species can colonize and infect all types of inserted and implanted medical devices. Infec- tions with this CONS is usually seen in hospitalized, elderly patients with indwelling Foley's catheters or other manipulations to the urinary system [2].

$S$. epidermidis is very common cause of foreign body related blood stream infections (FBR-BSI) [19]. Most of these infections are catheter-related blood stream infections (CRBSIs). FBR-BSI due CONS significantly increases ICU stay and health care cost [19]. These infections are associated with higher mortality rates. S. epidermidis is the most frequent cause of early prosthetic valve infective endocarditis (PVIE) [19]. Clinical course of PVIE due to $S$. epidermidis may be subacute or chronic, subtle and without fulminant signs of infections. It is the important cause of septicemia in febrile patients [19].

S. haemolyticus is associated in native valve endocarditis, septicemia, urinary tract infection (UTI), peritonitis, wound infections and infections of bone and joint $[18,4]$.

S. saprophyticusis an important cause acute urethritis. This pathogen can also cause infections known for $S$. epidermidis. Approximately 10 to $11 \%$ of all UTI are caused by $S$. saprophyticus, especially in sexually active females $[18,4]$. At times, $S$. saprophyticus UTI may be accompanied by BSI by same organism. In males, $S$. saprophyticus causes urethritis, epididymitis and chronic prostatitis [20]. Acute pyelonephritis, recurrent infection, nephrolithiasis, septicaemia and endocarditis are rarely seen as complications of S. saprophyticus [20].

S. lugdunensis is associated with arthritis, catheter related and urinary tract infections, bacteremia, prosthetic joint infections and endocarditis [18,4]. The course of disease of infective endocarditis due to S. lugdunensisis often destructive and unusually fulminant for a CONS species and is associated with high mortality rates [2]. Heart failure, abscess formation and embolism are frequent complications of $S$. lugdunensis infective endocarditis [21].

In recent years, there is alarming increase in the incidence of skin and soft tissue infections (SSTIs) due to $S$. lugdunensis [22]. S. lugdunensisinvolvement is also noted in perineal and inguinal abscess. Isolation of $S$. lugdunensis is also reported from brain abscess, peritonitis, osteomyelitis, discitis, septic arthritis in native joints [2]. S. lugdunensis is also known to cause complication of arthroscopy, endometritis with premature rupture of membranes, pyomyoma after cesarean section [2].

Virulence factors of CONS: Bacteria belonging to the genus Staphylococcus bear an ability to colonize and 
infect human host through armamentarium of multiple strategies that favor adherence/attachment, aggression, invasion, persistence and/or evasion of host's immune systems (both innate and acquired immunity) [2].

Although, this virulence mechanisms are well studied in S. aureus (the pervasive pathogen of the genus), there is dearth of information regarding virulence traits of CONS (the emerging pathogen).

The pathogenicity of CONS can be attributed to virulence factor involved in colonization rather than those responsible for aggression [2]. Adherence/attachment to the host tissue is the primary and most critical step in initiation of colonization and infection. Adhesion to host tissue confers certain properties to the colonizing or infecting strain. It prevents or at least reduces extent of microbial clearance by the host's defense mechanisms. Adherence also ensures delivery of toxins and enzymes to the target host cells.

The process of adherence also plays an important role in biofilm or slime formation on to the surface of indwelling or implanted medical devices. Biofilm formation is an important virulence trait as it protects the bacterial strain from the action of antimicrobial agents and the host's immune system [23]. Biofilm forming CONS isolates also demonstrate high resistance to methicillin. Koksal and associates (2007) reported significantly high methicillin resistance in biofilm forming CONS isolates compared to non-biofilm producers [24]. Various mechanisms like binding of antibiotics to contents of biofilm, decreased antibiotic penetration, slow growth of microorganism within the biofilm, altered gene expression in bacteria present in the biofilm and high bacterial density are identified for reduced antibiotic susceptibility in biofilm forming bacterial isolates [2].

In Staphylococcus various proteinaceous and nonproteinaceous adhesins are responsible for the process of adhesion [25]. Proteinaceous adhesins include covalently surface-anchored (CWA, SdrG/Fbe, SesC and Aap) and noncovalently surface-associated proteins that include autolysin/adhesion family (Atl), and membranespanning proteins [2]. The most important adhesin is AtlE, from $S$. epidermidis. It is similar to Atl of S. aureus and initiates adhesion of bacterial cells to the surface of polymer [2]. In recent years, homologous Atl proteins with same functions are also reported from other CONS such as $S$. caprae (AtlC), S. saprophyticus (Aas), $S$. lugdunensis (AtlL) and $S$. warneri $\mathrm{M}\left(\mathrm{Atl}_{\mathrm{WM}}\right)$. Polysaccharide intercellular adhesion (PIA) also known as poly$\mathrm{N}$-acetylglucosamine (PNAG), teichoic and lipoteichoic acids are examples of non-proteinaceous adhesins [2].

Various methods can be employed for detection of bio- film formation in CONS. These include Christensen method, brain heart infusion with sucrose and congo red agar method. Freeman et al (1989) reported congo red agar method to be cost effective, rapid, sensitive and reliable for detection of biofilm formation in CONS [26]. Tissue culture plate method proposed by Christensen et al (1986) is considered as the gold standard method for detection of biofilm formation [27].

Cell wall components of CONS also interfere with host's immune system. Mattsson and coworkers (1993) in their experiment proved the role of peptidoglycan and teichoic acid of $\mathrm{S}$. epidermidis in stimulating human monocytes to release tumor necrosis factor (TNF) $\alpha$, interleukin (IL) 1 , IL- $\beta$ and IL-1 [28].

CONS produce a variety of exoenzymes and proteins such as lipases, proteinases, esterases, phospholipases haemolysins and other toxins. These extracellular enzymes aid bacteria in establishment and progression of infection and to evade host defense mechanisms [2]. Among various exoenzymes, proteinases play an important role in pathogenicity of CONS. It inactivates host immune defenses by inactivating immunoglobulins, complement proteins, platelet microbicidal proteins and human antimicrobial peptides [2]. Proteinases also degrade elastin, fibrinogen and fibronectin and facilitate CONS to colonize and infect host's tissue [29].

Lantibiotics are produced by various CONS spp. like $S$. epidermidis (epidermin and Pep5), S. gallinarum (gallidermin), S. hominis (hominicin) and S. warneri (nukacin) [30]. L antibiotics are antibiotic-like peptides that also fulfill the criteria for bacteriocins. These L antibiotics play a putative role in bacterial interference on skin and mucus membranes and create an ecological niche for CONS [30]. Virulence factors like clumping factors and production of a thermostable DNAse are expressed in CONS spp. like $S$. lugdunensis and $S$. Schleiferi [30].

Laboratory diagnosis: As CONS are easily obtained from clinical material of most of infectious sites and are relatively resistant to drying and moderate changes in temperature, their isolation and cultivation is comparatively simple and uncomplicated [2]. However, like for any other pathogenic bacteria, the general principles of collection, transport and storage of clinical specimens are also applicable to CONS.

Precautionary measures should be followed while collecting specimen to avoid contamination with normal flora. Clinical specimens should be obtained from the focus or foci of infection. The sample is collected as per the nature of the lesions. Pus and wound swabs are collected from suppurative lesions. Sputum in collected in 
case of respiratory infections. Mid-stream urine is obtained whenever urinary tract infection is suspected. Blood cultures are collected for detecting bacteremia.

The standard protocol for laboratory diagnosis of CONS infections includes isolation of CONS on appropriate media followed by species identification by battery of biochemical reactions. Various commercially available kit-based systems can be also utilized for species identification. In recent years, with the advancement in molecular biology and its application in the field of medicine, conventional identification methods are largely replaced by advanced techniques like matrix-associated laser desorption ionization-time of flight mass spectrometry (MALDI-TOF MS) [2].

Direct microscopic method involves examination of Gram stained smear prepared from clinical specimens. Direct microscopy is of great value in case of examination of normally sterile fluids like cerebrospinal fluid and joint aspirate. In case of non-sterile fluids direct microscopic examination can be used for demonstration of inflammatory cells. On Gram staining, CONS appear as Gram-positive cocci usually arranged in pairs or tetrads but at times they appear as single, in clusters or in short chains of about three or four cells [2].

Being a non-fastidious organism, CONS grow well on most of microbiological media used routinely for isolation of bacteria in clinical laboratory services. Columbia or tryptic soya $5 \%$ de-fibrinated sheep or horse blood agar is used as primary isolation media of CONS regardless of specimen source [2]. Selective or enrichment media may be necessary for isolation of CONS from certain specimens like sputum and stool where other bacteria may be predominant. Enrichment media like dextrose broth increases the rate of isolation of CONS. Mannitol salt agar and phenylethyl alcohol agar are examples of selective media used for CONS. Generally, CONS grow within $18-24 \mathrm{~h}$ of incubation at $37^{\circ} \mathrm{C}$ (range $12-44^{\circ} \mathrm{C}$ ).

CONS colonies are nonpigmented, smooth with entire margin, glistening and opaque. However certain CONS species like $S$. chromogenes, $S$. devriesei, S. lugdunensis, S. sciuri, S. warneri and $S$. xylosusproduce grey yellow, yellow, or yellow orange pigmented colonies. $S$. haemolyticus and $S$. lugdunensis colonies are $\beta$ haemolytic [2].

"Small colony variant" (SCV) colonies are pinpoint and only $10 \%$ of the size of wild-type colonies [31]. These colonies show minimal or no haemolysis. SCV can be reverted back to wild type by growing the isolates in the presence of certain components like hemin, menadione and thymidine, and/or in capnophilic condition [16].
Like other members of Genus Staphylococci, CONS are catalase positive. Catalase test is using to differentiate family Micrococcaceae from family Streptococcaceae. Staphylococci contain cytochrome oxidase responsible for catalase positivity. As slide coagulase test may give false positive results in some CONS isolates, tube coagulase test is the traditional biochemical test used for differentiation of S. aureus and CONS [2]. CONS are coagulase negative. Stapylase test, Staphy slide, Staphaurrex and Slidex Staph kit are examples rapid latex-based test used as alternatives to conventional coagulase test [32].

Novobiocin resistance testing is used for speciation of CONS isolated from urine specimens (Hebert et al 1988). This test is performed on Mueller-Hinton agar or tryptic soy blood agar using $5 \mu$ gnovobiocin disc. Novobiocin resistance testing is used to distinguish $S$. saprophyticus from $S$. epidermidis.

Automated commercial identification systems for CONS include API Staph and ID32 Staph strips (bioMèrieux), the Rapidec Staph (bioMèrieux) system, Vitek 2 (bioMèrieux), the PosID Panel family (Siemens Healthcare Diagnostics, Deerfield, IL), the BBL Crystral identification system's Rapid Gram-Positive ID kit (BD Diagnostic Systems, Sparks, MD), the Phoenix automated microbiology system (BD Diagnostic Systems), and the Biolog systems (Biolog, Hayward, CA) [16]. The specificity of these kits is reported to be 70 to $>90 \%$ [16].

In recent years, molecular approaches are gaining importance for identification of CONS. Several nucleic acid-based methods are designed for CONS identification. However, these molecular systems cannot be used for detection of CONS directly from clinical specimens (except for Polymerase chain reaction (PCR)-based detection method such as Light Cycler Septi Fast test that detects CONS directly from blood sample) (Niederbrachtet al 2013). Varieties of nucleic acidbased system are available for identification of CONS isolated in culture. These include methods based on procedures of nucleic acid amplification, hybridization and sequencing [16].

Ribosomal genes, the glyceraldehydes-3-phosphate dehydrogenase-encoding ( $g a p$ ) gene, the gyrase ( gyr A) gene, the manganese-polymerase beta subunit (rpo $\beta$ ) gene, the elongation factor TU (tuf) gene, and $60-\mathrm{kDa}$ heat shock protein (HSP60/GroE) gene are examples of universal targets used for identification of CONS by PCR [16].

Peptide nucleic acid fluorescence in situ hybridization (PNA FISH) is used for rapid identification of CONS 
from positive blood culture smears (Hensley et al 2009). MALDI -TOF MS is recently identified as a sensitive and specific molecular technique for identification of CONS species (Clark et al 2013).

Antibiotic resistance.

As many of the CONS species often demonstrate decreased susceptibility and/or resistant to various classes of antibacterial drugs, their identification along with antibiogram plays a very important role in the selection of most appropriate and effective antibiotic for prophylaxis and treatment.

Various researchers from different parts of the world have highlighted the importance of different techniques for determining antibiotic resistance in CONS species. Woods and colleagues (1986) recommended the $24 \mathrm{~h}$ oxacillin disc diffusion test for detection of methicillin resistance in S. epidermidis [33]. Researchers like Archer et al (1990), Ubukata et al (1990) and Suzuki et al (1992) highlighted importance of molecular techniques like PCR and the mecA gene probe for detection of methicillin resistance $[34,35,36]$.

Nearly, $80-90 \%$ of clinical CONS isolates produces inducible $\beta$-lactamase [37]. Production of low-affinity penicillin-binding protein (PBP2a) is the most important mechanism known for methicillin resistance [7]. PBP2a is seen in CONS species like, S. epidermidis, S. haemolyticus, S. hominis, S. simulans, S. saprophyicus, S. sciuri, S. capitis, S. caprae and S. warneri. Methicillin resistant strains are multi-resistant as they demonstrate resistance to various classes of antibacterial agents besides beta-lactams drugs [7].

Clinical strains of $S$. haemolyticus demonstrate decreased susceptibility to vancomycin. Strains of $S$. haemolyticus and $S$. epidermidis also demonstrate decreased susceptibility to other glycopeptides and teicoplanin [3]. Acquired glycopeptide resistance was first detected in CONS [38]. The mechanism responsible for glycopeptide resistance is multifactorial and is expression related. Glycopeptide resistance can be acquired by exposure of CONS strains to teicoplanin and vancomycin [38].

Antibiotic resistance in CONS varies as per species. $S$. haemolyticus, $S$. epidermidis and $S$. hominis demonstrate high resistance rates. Studies have reported 76$96 \%$ oxacillin resistance, $80-90 \%$ erythromycin resistance and $26-29 \%$ teicoplaninnonsusceptibility in S. haemolyticus [4].

Gatermann et al (2007) reported oxacillin resistance in $80 \%$ of $S$. hominis isolates. Various researchers have reported oxacillin resistance in $38-81 \%$ of $S$. epidermid- is. S. lugdunensis often demonstrates uniform in vitro susceptibility to most antibiotic classes [39]. Most CONS species demonstrate $100 \%$ susceptibility to novel antibiotics like linezolid, daptomycin, tigecycline and quinupristin or dalfopristin [4].

\section{CONCLUSION}

In recent years, coagulase negative Staphylococci (CONS) are increasingly recognized as one of the major causes of health care associated infections (HCAI). CONS infections are one of the major causes of morbidity and mortality in hospitalized patients. As many of the CONS species often demonstrate decreased susceptibility and/or resistant to various classes of antibacterial drugs, their identification along with antibiogram plays a very important role in the selection of most appropriate and effective antibiotic for prophylaxis and treatment.

\section{REFERENCES}

[1] Szymanska G, Szemraj M, Szewczyk E. 2011. Species-specific sensitivity of coagulase-negative staphylococci to single antibiotics and their combinations. Pol J Microbiol. 2011;60:155-61.

[2] Becker K, Heilmann C, Peters G. CoagulaseNegative Staphylococci. ClinMicrobiol Rev. 2014;27:870-26

[3] Kloos W, Bannerman T. 1994. Update on clinical significance of coagulase-negative staphylococci. ClinMicrobiol Rev.1994;7:117-40

[4] Piette A, Verschraegen G. Role of coagulasenegative staphylococci human disease. Vet Microbiol. 2009; 134: 45

[5] Schaberg D, Culver D, Gaines R. Major trends in the microbial etiology of nosocomial infection. Am J Med. 1991:725-735.

[6] Archer G. Alteration of cutaneous staphylococcal flora as a consequence of antimicrobial prophylaxis. Rev Infect Dis. 1991;13:5805-9

[7] Archer G, Climo M. Antimicrobial susceptibility of coagulase negative Staphylococci. Antimicrob agents Chemother. 1994;38:2231-4

[8] Huebner J, Goldmann D. Coagulase negative staphylococci: Role as Pathogens. Annu Rev Med. 1999;50:223-36

[9] Evans C, Smith W, Johnston E, Giblett E. Bacterial flora of the normal human skin. J Invest Dermatol. $1950 ; 15: 305-24$.

[10] Willcox M. Characterization of the normal microbiota of the ocular surface. Exp Eye Res. 2013;117:99-105.

[11] Von Eiff C, Proctor R, Peter G. Coagulasenegative staphylococci: pathogens have major role in nosocomial infections. Postgrad Med.2001;110:63-76. 
[12] Baird Parker A. A Classification of Micrococci and Staphylococci based on Physiological and Biochemical tests. J Gen Microbiol. 1963;30:40927.

[13] Heikens E, Fleer A, Paauw A, Florijn A, Fluit A Comparison of genotypic and phenotypic methods for species-level identification of clinical isolates of coagulase-negative staphylococci. J. Clin. Microbiol. 2005;43:2286-90.

[14] Layer F, Ghebremedhin B, Moder K, König W, König B. Comparative study using various methods for identification of Staphylococcus species in clinical specimens. J.Clin. Microbiol. 2006;44:2824-30.

[15] Mellmann A, Becker K, Von Eiff C, Keckevoet U, Schumann P, Harmsen D. Sequencing and staphylococci identification. Emerg. Infect. Dis. 2006; $12: 333-6$

[16] Becker K, Laham NA, Fegeler W, Proctor RA, Peters G, Von Eiff C. Fourier-transform infrared spectroscopic analysis is a powerful tool for studying the dynamic changes in Staphylococcus aureussmall colony variants. J ClinMicrobiol. 2006; $44: 3274-8$

[17] Koneman E, Allen S, Janda W, Schreckenberger P. The gram positive cocci Part I: Staphylococci and related organism In colour Atlas and Textbook of Diagnostic Microbiology $1997 ; 5^{\text {th }}$ Ed Lippincott publishers

[18] Bannerman T. In Murray P, Baron E, Jorgerson J, Pfaller M, Yolken R (Eds.) Staphylococcus, Micrococcus and other catalase-positive cocci that grow aerobically. Manual of clinical microbiology, ASM Press, Washington. 2003

[19] Sievert D, Ricks P, Edwards J, Schneider A, Patel J, SrinivasAet al. Antimicrobial-resistant pathogens associated with healthcare-associated infections: summary of data reported to the National Healthcare Safety Network at the Centers for Disease Control and Prevention, 2009-2010. Infect Control HospEpidemiol. 2013;34:1-14

[20] Raz R, Colodner R, Kunin C. Who are youStaphylococcus saprophyticus? Clin Infect Dis. 2005;40:896-8

[21] Anguera I, Del Rio A, Miro J, Matinez-Lacasa X, Marco F, Guma J et al. Staphylococcus lugdunensis infective endocarditis: description of 10 cases and analysis of native valve, prosthetic valve, and pacemaker lead endocarditis clinical profiles. Heart. 2005;91:e10.

[22] Bocher S, Tonning B, Skow R, Prag J. Staphylococcus lugdunensis, a common cause of skin and soft tissue infections in the community. J ClinMicrobiol. 2009; 47:946-50

[23] Mack D. Molecular mechanism of S. epidermidisbiofilm formation. J Hosp Infect,1999; 43: S113-25

[24] Koksal F, Yasar H, Samasti M. Antibiotic resistance patterns of coagulase-negative Staphylo- coccus strains isolated from blood cultures of septicemic patients in Turkey. Microbiol Res. 2007;164(4):404-10

[25] Heilmann C. Adhesion mechanisms of staphylococci. AdvExp Med Biol. 2011;715:105-23

[26] Freeman D, Falkiner F, Keane C. New method for detection of slime production by coagulase negative staphylococci. J ClinPathol. 1989;42:872-4

[27] Christensen G, Simposon W, Younger J, Baddour F, Barrett D, Melton D et al. Adherence of coagulase-negative staphylococci to plastic tissue culture plates: a quantitative model for the adherence of staphylococci to medical devices. J ClinMicrobiol. 1986;22:996-1006.

[28] Mattsson E, VerhageL,Rollof J, Fleer A, Verhoef J, Van Dijk H.Peptidoglycan and teichoic acid from Staphylococcus epidermidisstimulate human monocytes to release tumour necrosis factor- $\alpha$, interleukin-1 and interleukin-6. FEMS Immunol. Med. Microbiol. 1993;7:281-7

[29] Mack D, Fischer W, Krokotsch A, Leopold K, Hartmann R, Egge H, Laufs R.1996. The intercellular adhesin involved in biofilm accumulation of Staphylococcus epidermidisis a linear beta-1,6linked glucosaminoglycan: purification and structural analysis. J. Bacteriol. 1996;178: 175-83

[30] Gotz F, Perconti S, Popella P, Werner R, SchlagM.Epidermin and gallidermin: staphylococcal lantibiotics. Int. J. Med. Microbiol. 2014;304: 63-71

[31] Proctor RA, von Eiff C, Kahl BC, Becker K, McNamara P, Herrmann M, Peters G. Small colony variants: a pathogenic form of bacteria that facilitates persistent and recurrent infections. Nat. Rev. Microbiol.2006;4:295-305

[32] Baird D. Staphylococcus: Cluster forming Gram positive cocci In; Mackie McCartney Practical Medical Microbiology $14^{\text {th }}$ edition Collee J, Fraser A, Marmion B, Simmon A Chruchill Livingstone, New York. 2006;245-62

[33] Woods G, Hall G, Rutherford I, Pratt K, Knapp C. 1986. Detection of methicillin-resistant Staphylococcus epidermidis. J. Clin. Microbiol. $1986 ; 24: 349-52$

[34] Archer G, Pennell E. Detection of methicillin resistance in staphylococci by using a DNA probe. Antimicrob Agents Chemother. 1990;34:2500-4

[35] UbukataK,Nonoguchi R, Song M, Matsuhashi M, Konno M. Homology of mecA gene in methicillinresistantStaphylococcushaemolyticus and Staphylococcussimulans to that of Staphylococcus aureus. Antimicrob.Agents Chemother. 1990;34:170-2.

[36] Suzuki E, Hiramatsu K, Yokota T.Survey of methicillin-resistant clinical strains of coagulasenegative staphylococci for mecA gene distribution. Antimicrob Agents Chemother 1992;36:429-34.

[37] Archer G, Scott J. Conjugative transfer genes in 
staphylococcus isolates from the United States. Antimicrob agents Chemother. 1991;35:2500-4

[38] Biavasco F, Giovanetti E, Montanari M, Lupidi R, Varaldo P. 1991. Development of in-vitro resistance to glycopeptides antibiotics: assessment in staphylococci of different species. J. Antimicrob. Chemother. 1991;27:71-9

[39] Baird Parker A. A Classification of Micrococci and Staphylococci based on Physiological and Biochemical tests. J Gen Microbiol. 1963;30:40927. 